\title{
A Review on the Bound-State Solutions of the Stationary Schrödinger Equation for General Pseudo-Coulomb Potential
}

\author{
Spiros Konstantogiannis \\ 4 Antigonis Street, Nikaia 18454, Athens, Greece \\ Email: spiroskonstantogiannis@gmail.com
}

\begin{abstract}
Considering the stationary Schrödinger equation for a general pseudo-Coulomb potential as the normal form of the associated Laguerre equation, we review, in one and three dimensions, the bound-state solutions for the potential, when the inverse-square-term coupling is not less than a negative critical value. We show that, as a consequence of the inverse-square-term coupling being a two-to-one mapping for all but one of the allowed negative values of its parameter, an additional sequence of bound-state energies emerges for each of the respective potentials. In this framework, the slightest relaxation of the boundary condition for the radial wave function at the origin results in minus-infinity ground-state energy for the Coulomb potential, rendering the hydrogen atom unstable.
\end{abstract}

Keywords: associated Laguerre equation; pseudo-Coulomb potential; inverse square potential; Kratzer potential; valence electron model; additional energies; instability.

\section{Introduction}

We examine a general pseudo-Coulomb potential containing an attractive Coulomb term and an inverse square term with a coupling that is not less than a negative critical value, below which a "fall" of the particle to the origin takes place [1]. Our goal is to present, in a uniform way, the bound-state solutions for the potential in the whole domain of the inverse-square-term coupling. We also wish to highlight the emergence of additional energies, as a consequence of the inverse-square-term coupling being a two-toone mapping in a subset of its domain, and establish a relation between the boundary condition for the radial wave function at the origin and the stability of the hydrogen atom.

The general pseudo-Coulomb potential we consider finds applications in many fields of physics. When its inverse square term is repulsive, the potential is known as the Kratzer potential, one of the most important model interactions in quantum physics, which was first introduced to describe the vibrationrotation spectra of diatomic molecules [2]. Since then, it has been widely used to describe molecular structures and interactions [3], and nowadays it appears in different fields of physics and chemistry, such as chemical physics, quantum chemistry, molecular physics, nuclear physics, and thermodynamics. When the inverse square term of the potential vanishes, the potential reduces to an attractive Coulomb potential that can be used to describe the motion of the electron in the hydrogen atom, an elementary system of paramount importance in quantum physics. Finally, pseudo-Coulomb potentials with weakly attractive inverse square terms are used as molecular potentials for the description of the spectra of alkali metals $[4,5]$.

The rest of the paper is organized as follows: in section 2, we consider the one-dimensional stationary Schrödinger equation for a general pseudo-Coulomb potential as the normal form of the associated Laguerre equation and, by means of two theorems listed in the appendix, we derive the general form of the bound-state solutions for the potential in terms of terminating Frobenius solutions of the associated Laguerre equation, and determine the domain of the inverse-square-term coupling and its minimum negative value. In section 3 , we observe that the inverse-square-term coupling is a two-to-one mapping in $(-1,0) \backslash\{-1 / 2\}$ and, as a consequence, each of the respective potentials, which all have attractive inverse square term, has an additional sequence of bound-state energies. In section 4 , we examine the potential in three dimensions and observe that if the particle has non-zero orbital angular momentum, there exists an interval of negative values in the domain of the inverse-square-term coupling for which the respective potential, which has attractive inverse square term, has no additional energies. In section 5 , we examine the case where the inverse square term is repulsive and the resulting potential is the 
Kratzer potential, while in section 6, we examine the case where the inverse square term is weakly attractive and the resulting potential is used as a valence electron model. In section 7, we establish a connection between the boundary condition for the radial wave function at the origin and the stability of the hydrogen atom, by showing that the slightest relaxation in the boundary condition results in the emergence of minus-infinity ground-state energy for the resulting Coulomb potential. Finally, in section 8, we summarize and conclude. In the appendix, we list the two theorems we use in section 2.

\section{The One-Dimensional Case}

Assuming that for $x \leq 0$, the potential is $+\infty$, we wish to solve the stationary Schrödinger equation on the half-line $x>0$ for a general pseudo-Coulomb potential of the form

$$
V(x)=-\frac{a}{x}+\frac{b}{x^{2}}
$$

where the coupling $a$ is positive, while the coupling $b$ is positive, zero, or negative down to a critical value.

The stationary Schrödinger equation for a potential of the above form is the normal form of the associated Laguerre equation

$$
x y^{\prime \prime}(x)+(v+1-x) y^{\prime}(x)+\lambda y(x)=0, \quad v, \lambda \in \mathbb{R}
$$

in the region $x>0$, since if

$$
y(x)=x^{-(v+1) / 2} z(x) \exp \left(\frac{x}{2}\right),
$$

the associated Laguerre equation takes its normal form

$$
z^{\prime \prime}(x)+\left(-\frac{1}{4}+\frac{2 \lambda+v+1}{2 x}+\frac{1-v^{2}}{4 x^{2}}\right) z(x)=0
$$

To compare (1) with the stationary Schrödinger equation

$$
\psi^{\prime \prime}(x)+\frac{2 m}{\hbar^{2}}(E-V(x)) \psi(x)=0,
$$

where $E$ is the energy of the eigenstate described by the wave function $\psi(x)$ for a potential $V(x), m$ is the mass of the examined particle, and $\hbar$ is the reduced Planck constant, we'll write the stationary Schrödinger equation in dimensionless form. To this end, we choose a positive length parameter $l_{0}$ and set

$$
x \rightarrow l_{0} x, E \rightarrow \frac{\hbar^{2}}{2 m l_{0}^{2}} E, \quad V(x) \rightarrow \frac{\hbar^{2}}{2 m l_{0}^{2}} V(x) .
$$

Then, the stationary Schrödinger equation reads, in dimensionless form,

$$
\psi^{\prime \prime}(x)+(E-V(x)) \psi(x)=0
$$

Assuming that the normalization constant of the wave function carries its dimensions, then omitting the normalization constant makes the wave function also dimensionless. Then, comparing (2) with (1) yields

$$
\begin{gathered}
E=-\frac{1}{4} \\
V(x)=-\frac{2 \lambda+v+1}{2 x}-\frac{1-v^{2}}{4 x^{2}}
\end{gathered}
$$

The dimensionless wave function $\psi(x)$ then takes the place of the function $z(x)$ in $(1)$, and thus

$$
\psi(x)=x^{(v+1) / 2} y(x) \exp \left(-\frac{x}{2}\right)
$$

The function $y(x)$ is solution to the associated Laguerre equation. To restore the dimensions, we do the inverse substitutions, i.e. 


$$
x \rightarrow \frac{1}{l_{0}} x, E \rightarrow \frac{2 m l_{0}^{2}}{\hbar^{2}} E, V(x) \rightarrow \frac{2 m l_{0}^{2}}{\hbar^{2}} V(x),
$$

and then the energy (3) and the potential (4) become, respectively,

$$
\begin{gathered}
E=-\frac{\hbar^{2}}{8 m l_{0}^{2}} \\
V(x)=-\frac{(2 \lambda+v+1) \hbar^{2}}{4 m l_{0} x}-\frac{\left(1-v^{2}\right) \hbar^{2}}{8 m x^{2}}
\end{gathered}
$$

In dimensional form, the wave function (5) is written as

$$
\psi(x)=A\left(\frac{x}{l_{0}}\right)^{(v+1) / 2} y\left(\frac{x}{l_{0}}\right) \exp \left(-\frac{x}{2 l_{0}}\right)
$$

where the normalization constant $A$ carries the dimensions of the wave function. This is the expression of the wave function in the region $x>0$. In the region $x \leq 0$, the wave function vanishes, and since it must be continuous at zero,

$$
\psi(0)=0
$$

Moreover, since we seek bound-state solutions, the wave function (8) must be square-integrable in $[0, \infty)$. On the other hand, the function $y\left(x / l_{0}\right)$ in $(8)$ is a solution to the associated Laguerre equation which, depending on the value of the parameter $v$, has one or two Frobenius series solutions [6]. In line with the theorem 1 of the appendix, the non-terminating Frobenius solutions of the associated Laguerre equation behave as $\exp (x)$ at infinity, thus if such a solution takes the place of $y\left(x / l_{0}\right)$ in (8), the resulting wave function behaves as $\exp \left(x / 2 l_{0}\right)$ at infinity, and then it is not square integrable. In the case where the associated Laguerre equation has only one Frobenius solution, the second linearly independent solution has a logarithmic part [6] endowing the wave function with a zero at $l_{0}$, which is physically unjustifiable with respect to the potential (7). Thus, only terminating Frobenius solutions to the associated Laguerre equation can take the place of $y\left(x / l_{0}\right)$ in (8). Then, in line with the theorem 2 of the appendix, we have the following cases:

i. If $v$ is not integer and $\lambda$ is non-negative integer, i.e. $\lambda=0,1, \ldots$, we have a polynomial solution $y\left(x / l_{0}\right)$ of degree $\lambda$ with non-zero constant term, thus $y(0) \neq 0$, and the boundary condition (9) is satisfied only when $v>-1$. Then $v+1>0$ and since $\lambda$ is non-negative, the Coulomb term of the potential (7) is attractive, while the inverse square term is attractive if $-1<v<1$ and repulsive if $v>1$. Since $v$ is not integer, in this case the inverse square term cannot vanish.

For $v$ fixed, keeping also $l_{0}$ fixed, the energy (6) is also fixed, while the Coulomb term of the potential changes with $\lambda$ and the inverse square term is fixed. Then, we obtain bound-state solutions of pseudoCoulomb potentials having the same inverse square term and different Coulomb terms, with the same energy and wave functions being given by the same exponential factor and polynomials $y\left(x / l_{0}\right)$ of different degree $\lambda$. This is similar to the so-called second type of quasi-exact solvability, which is described in [7], but we don't examine such models here.

On the other hand, for $v$ fixed, the Coulomb term is fixed if $(2 \lambda+v+1) / l_{0}$ is fixed, and since $\lambda$ changes, $l_{0}$ changes too. Then, setting

$$
l_{0}=(2 \lambda+v+1) \tilde{l}_{0}
$$

with $\tilde{l}_{0}$ being a new, fixed positive constant with dimensions of length, we rewrite (10) as

$$
l_{0}=2(n+\tilde{v}) \tilde{l}_{0}
$$

where

$$
\begin{aligned}
& n=\lambda+1 \\
& \tilde{v}=\frac{v-1}{2}
\end{aligned}
$$

where $n=1,2, \ldots$ Since $v>-1$, then $\tilde{v}>-1$ too. Also, since $v$ is not integer, $v-1$ is not integer either, thus $\tilde{v}$ is not integer and not half-integer.

By means of (11), the energy (6) takes the form 


$$
E_{n \tilde{v}}=-\frac{\varepsilon_{0}}{(n+\tilde{v})^{2}}
$$

where

$$
\varepsilon_{0}=\frac{\hbar^{2}}{32 m \tilde{l}_{0}^{2}}
$$

The constant $\varepsilon_{0}$ is positive and has dimensions of energy. Next, using (10) and that $v=2 \tilde{v}+1$, the potential (7) becomes

$$
V_{\tilde{v}}(x)=-\frac{\hbar^{2}}{4 m \tilde{l}_{0} x}+\frac{\tilde{v}(\tilde{v}+1) \hbar^{2}}{2 m x^{2}}
$$

Using (15), we replace $\hbar^{2} / m$ with $32 \varepsilon_{0} \tilde{l}_{0}^{2}$, ending up to

$$
V_{\tilde{v}}(x)=-\frac{8 \varepsilon_{0} \tilde{l}_{0}}{x}+\frac{16 \tilde{v}(\tilde{v}+1) \varepsilon_{0} \tilde{l}_{0}^{2}}{x^{2}}
$$

Since $\tilde{v}+1>0$, then for $-1<\tilde{v}<0$, the inverse square term is attractive, while for $\tilde{v}>0$, it is repulsive.

Finally, using (11) and that $v=2 \tilde{v}+1$, the wave function (8) reads

$$
\psi_{n \tilde{v}}(x)=A_{n \tilde{v}}\left(\frac{x}{2(n+\tilde{v}) \tilde{l}_{0}}\right)^{\tilde{v}+1} P_{n-1}\left(\frac{x}{2(n+\tilde{v}) \tilde{l}_{0}}\right) \exp \left(-\frac{x}{4(n+\tilde{v}) \tilde{l}_{0}}\right)
$$

The polynomial $P_{n-1}$ is of degree $\lambda=n-1$ and, in line with the theorem 2 of the appendix, it is given by $^{1}$

$$
P_{n-1}\left(\frac{x}{2(n+\tilde{v}) \tilde{l}_{0}}\right)=\sum_{k=0}^{n-1} c_{k}\left(\frac{x}{2(n+\tilde{v}) \tilde{l}_{0}}\right)^{k}, c_{k+1}=\frac{k-(n-1)}{(k+2 \tilde{v}+2)(k+1)} c_{k}, k=0,1, \ldots, n-2
$$

If $n=1$, the polynomial $P_{n-1}$ is a non-zero constant and can be incorporated into the normalization constant of the wave function (17). Then, for $n=1,2, \ldots,(14)$ and (17) give, respectively, the boundstate energies and the wave functions of the pseudo-Coulomb potential (16) in the positive region. We also note that in (14), (16), and (17), we have expressed the energy, the potential, and the wave function in terms of the new parameters $\tilde{v}$ and $n$, instead of the initial ones $v$ and $\lambda$.

ii. If $v$ is not integer and $\lambda=-v,-v+1$,..., i.e. $\lambda=-v+n-1$ with $n=1,2, \ldots$, we have a nonpolynomial terminating series solution $y\left(x / l_{0}\right)$ which, in the region $x>0$, is written as the product of $\left(x / l_{0}\right)^{-v}$ and a polynomial of degree $\lambda+v=n-1$. Then, the wave function (8) takes the form

$$
\psi_{n v}(x)=A_{n v}\left(\frac{x}{l_{0}}\right)^{(1-v) / 2} P_{n-1}\left(\frac{x}{l_{0}}\right) \exp \left(-\frac{x}{2 l_{0}}\right)
$$

In line with the theorem 2 of the appendix, the constant term of the polynomial $P_{n-1}$ does not vanish, i.e. $P_{n-1}(0) \neq 0$, and thus for the boundary condition (9) to hold, $v<1$. Then, using that $\lambda=n-(v+1)$ and that $n \geq 1$, we easily see that the Coulomb term of the potential (7) is again attractive. Then, following the same steps as in the previous case, we introduce the parameter $\tilde{v}=-(v+1) / 2$ and write the energy (6), the potential (7), and the wave function (18) in the form of (14), (16), and (17), respectively, where, in line with the theorem 2 of the appendix, the polynomial $P_{n-1}$ is defined, in terms of the parameters $\tilde{v}$ and $n$, by the same recurrence relation as in the previous case. Since $v<1$, then $\tilde{v}>-1$, and since $v$ is not integer, $-(v+1)$ is also not integer, thus $\tilde{v}$ is not integer and not halfinteger. Therefore, in terms of the parameters $\tilde{v}$ and $n$, this case is the same as the previous one.

iii. If $v$ and $\lambda$ are non-negative integers, i.e. if $v, \lambda=0,1, \ldots$, we have a polynomial solution $y\left(x / l_{0}\right)$ of degree $\lambda$, which is, up to a multiplicative real constant that can be incorporated into the normalization constant of the wave function (8), the associated Laguerre polynomial $L_{\lambda}^{\nu}(x)$ of degree $\lambda$ with parameter $v$. If $v=0$, the associated Laguerre polynomial reduces to the (simple) Laguerre polynomial

\footnotetext{
${ }^{1}$ Compared to the formula given in the appendix, we renamed the summation index to $k$ and used that $v=2 \tilde{v}+1$.
} 
$L_{\lambda}(x)$ of degree $\lambda$. Since $v \geq 0$, the wave function (8) vanishes at zero, thus the boundary condition (9) holds. Since $2 \lambda+v+1>0$, the Coulomb term of the potential (7) is again attractive, while the inverse square term is attractive if $v=0$, particularly it becomes most attractive for $v=0$, it vanishes if $v=1$, and it becomes repulsive if $v=2,3, \ldots$ We introduce again the constant $\tilde{l}_{0}$ through (10) which then takes the form of (11), with the parameters $n$ and $\tilde{v}$ being given by (12) and (13), respectively. Then, the energy (6) and the potential (7) take the same form as in case i, i.e. they are given by (14) and (16), respectively, and the wave function (8) takes the form

$$
\psi_{n \tilde{v}}(x)=A_{n \tilde{v}}\left(\frac{x}{2(n+\tilde{v}) \tilde{l}_{0}}\right)^{\tilde{v}+1} L_{n-1}^{2 \tilde{v}+1}\left(\frac{x}{2(n+\tilde{v}) \tilde{l}_{0}}\right) \exp \left(-\frac{x}{4(n+\tilde{v}) \tilde{l}_{0}}\right)
$$

Since $v=0,1, \ldots$, the parameter $\tilde{v}$ now takes the values $-1 / 2,0,1 / 2 \ldots$ Using the closed-form expression of the associated Laguerre polynomials [8], we have

$$
L_{n-1}^{2 \tilde{v}+1}\left(\frac{x}{2(n+\tilde{v}) \tilde{l}_{0}}\right)=\sum_{s=0}^{n-1} \frac{(-1)^{s}}{s !}\left(\begin{array}{c}
n+2 \tilde{v} \\
n-1-s
\end{array}\right)\left(\frac{x}{2(n+\tilde{v}) \tilde{l}_{0}}\right)^{s}
$$

Before proceeding to the next case, let us have a look at the subcases $\tilde{v}=-1 / 2$ and $\tilde{v}=0$.

For $\tilde{v}=-1 / 2$, the dimensionless coupling $\tilde{v}(\tilde{v}+1)$ of the inverse square term in (16) takes its minimum value, which is $-1 / 4$. This is the critical value of the inverse-square-term coupling, since for smaller values, the particle falls to the centre (see [1], where our dimensionless coupling $\tilde{v}(\tilde{v}+1)$ is denoted by $-\gamma$ ). In this subcase, the potential (16) reads

while its bound-state energies are, from (14),

$$
V(x)=-\frac{8 \varepsilon_{0} \tilde{l}_{0}}{x}-\frac{4 \varepsilon_{0} \tilde{l}_{0}^{2}}{x^{2}},
$$

$$
E_{n}=-\frac{\varepsilon_{0}}{\left(n-\frac{1}{2}\right)^{2}}, n=1,2, \ldots
$$

and the respective eigenfunctions, as given by (19), are

$$
\psi_{n}(x)=A_{n}\left(\frac{x}{2\left(n-\frac{1}{2}\right) \tilde{l}_{0}}\right)^{1 / 2} L_{n-1}\left(\frac{x}{2\left(n-\frac{1}{2}\right) \tilde{l}_{0}}\right) \exp \left(-\frac{x}{4\left(n-\frac{1}{2}\right) \tilde{l}_{0}}\right),
$$

since $L_{n-1}^{0}(x)=L_{n-1}(x)$.

For $\tilde{v}=0$, the inverse square term of the potential (16) vanishes and we are left only with the attractive Coulomb term, i.e.

$$
V(x)=-\frac{8 \varepsilon_{0} \tilde{l}_{0}}{x}, x>0
$$

This is a version of the one-dimensional hydrogen atom [9]. Its bound-state energies are given by (14) for $\tilde{v}=0$, i.e.

$$
E_{n}=-\frac{\varepsilon_{0}}{n^{2}}, n=1,2, \ldots
$$

with its respective eigenfunctions being, by means of (19),

$$
\psi_{n}(x)=A_{n} x L_{n-1}^{1}\left(\frac{x}{2 n \tilde{l}_{0}}\right) \exp \left(-\frac{x}{4 n \tilde{l}_{0}}\right)
$$

where we incorporated the $n$-dependent factor $1 / 2 n \tilde{l}_{0}$ into the normalization constant $A_{n}$.

iv. If $v$ is negative integer and $\lambda=-v,-v+1, \ldots$, we have a polynomial solution

$$
y\left(\frac{x}{l_{0}}\right)=c_{0}\left(\frac{x}{l_{0}}\right)^{-v} L_{n-1}^{-v}\left(\frac{x}{l_{0}}\right)
$$


where we set $\lambda+v=n-1$ with $n=1,2, \ldots$ Then, the wave function (8) takes the form, if we incorporate the constant $c_{0}$ into the normalization constant of the wave function,

$$
\psi_{n v}(x)=A_{n v}\left(\frac{x}{l_{0}}\right)^{(1-v) / 2} L_{n-1}^{-v}\left(\frac{x}{l_{0}}\right) \exp \left(-\frac{x}{2 l_{0}}\right)
$$

Since $v$ is negative integer, the wave function (21) vanishes at zero, thus the boundary condition (9) is satisfied. Moreover, since $\lambda=n-(v+1)$ and $n \geq 1$, the Coulomb term of the potential (7) is again attractive, while the inverse square term vanishes if $v=-1$ and it is repulsive if $v=-2,-3, \ldots$. As in the previous cases, we introduce again the constant $\tilde{l}_{0}$ through $(10)$ which, using that $\lambda=n-(v+1)$, takes the form of $(11)$, with $\tilde{v}=-(v+1) / 2$. Then, since $v$ is negative integer, $\tilde{v}=0,1 / 2,1 \ldots$, i.e. the parameter $\tilde{v}$ takes the values it takes in the previous case, except the value $-1 / 2$. In terms of the parameters $\tilde{v}$ and $n$, the energy (6) takes the form of (14), the potential (7) takes the form of (16), and the wave function (21) takes the form of (19). Therefore, in terms of the parameters $\tilde{v}$ and $n$, this case is covered by the previous one.

To summarize the cases $\mathrm{i}-\mathrm{iv}$, if $\tilde{v}>-1$, the bound-state energies of the general pseudo-Coulomb potential

are given by

$$
V_{\tilde{v}}(x)=-\frac{8 \varepsilon_{0} \tilde{l}_{0}}{x}+\frac{16 \tilde{v}(\tilde{v}+1) \varepsilon_{0} \tilde{l}_{0}^{2}}{x^{2}}
$$

$$
E_{n \tilde{v}}=-\frac{\varepsilon_{0}}{(n+\tilde{v})^{2}}
$$

with $n=1,2, \ldots, \varepsilon_{0}=\hbar^{2} / 32 m \tilde{l}_{0}^{2}$, and $\tilde{l}_{0}$ is a positive constant with dimensions of length.

a. If $\tilde{v} \neq-1 / 2,0,1 / 2, \ldots$, the energy eigenfunctions are given by

$$
\psi_{n \tilde{v}}(x)=A_{n \tilde{v}}\left(\frac{x}{2(n+\tilde{v}) \tilde{l}_{0}}\right)^{\tilde{v}+1} P_{n-1}\left(\frac{x}{2(n+\tilde{v}) \tilde{l}_{0}}\right) \exp \left(-\frac{x}{4(n+\tilde{v}) \tilde{l}_{0}}\right)
$$

where

$$
P_{n-1}\left(\frac{x}{2(n+\tilde{v}) \tilde{l}_{0}}\right)=\sum_{k=0}^{n-1} c_{k}\left(\frac{x}{2(n+\tilde{v}) \tilde{l}_{0}}\right)^{k}, c_{k+1}=\frac{k-(n-1)}{(k+2 \tilde{v}+2)(k+1)} c_{k}, k=0,1, \ldots, n-2
$$

If $n=1$, the polynomial $P_{n-1}$ is a non-zero constant.

b. If $\tilde{v}=-1 / 2,0,1 / 2 \ldots$, the energy eigenfunctions are given by

$$
\psi_{n \tilde{v}}(x)=A_{n \tilde{v}}\left(\frac{x}{2(n+\tilde{v}) \tilde{l}_{0}}\right)^{\tilde{v}+1} L_{n-1}^{2 \tilde{v}+1}\left(\frac{x}{2(n+\tilde{v}) \tilde{l}_{0}}\right) \exp \left(-\frac{x}{4(n+\tilde{v}) \tilde{l}_{0}}\right)
$$

where $L_{n-1}^{2 \tilde{v}+1}(x)$ is the associated Laguerre polynomial of degree $n-1$ with parameter $2 \tilde{v}+1$.

\section{Additional Energies When the Inverse Square Term Is Attractive}

The dimensionless coupling $\tilde{v}(\tilde{v}+1)$ of the inverse square term of the potential (22) is a quadratic polynomial in $\tilde{v}$, with a global minimum at $-1 / 2$, around which it is symmetric. However, as a consequence of the boundary condition (9), the domain of the coupling is not the whole $\mathbb{R}$, but the interval $(-1, \infty)$, and thus the coupling is symmetric only in $(-1,0)$. Then, for every $\tilde{v} \in(-1,0) \backslash\{-1 / 2\}$, the coupling is two-to-one, while for all other values, it is one-to-one. Particularly, as a function of $\tilde{v}$, the coupling $\tilde{v}(\tilde{v}+1)$ maps $(-1,-1 / 2]$ and $[-1 / 2,0)$ to $[-1 / 4,0)$, one time each, and $[0, \infty)$ to itself, one time too. Thus, the coupling takes all positive values, with each one of them corresponding to only one value of $\tilde{v}$, and thus the respective pseudo-Coulomb potentials (22), having repulsive inverse square terms, have only one sequence of bound-state energies (23). On the other hand, the coupling does not 
take all negative values, but only those belonging to $[-1 / 4,0)$, with each one of them, except $-1 / 4$, corresponding to two values of $\tilde{v}$, and thus the respective pseudo-Coulomb potentials (22), having attractive inverse square terms, have now two sequences of bound-state energies (23). For each of these potentials, the two values of $\tilde{v}$ are symmetric around $-1 / 2$, with one belonging to $(-1,-1 / 2)$ and the other to $(-1 / 2,0)$, thus introducing the local parameter $v^{\prime}$ such that $0<v^{\prime}<1 / 2$, the two sequences of bound-state energies (23) for each of the these potentials are respectively written as

$$
-\frac{\varepsilon_{0}}{\left(n-\frac{1}{2}-v^{\prime}\right)^{2}},-\frac{\varepsilon_{0}}{\left(n-\frac{1}{2}+v^{\prime}\right)^{2}} .
$$

The two sequences have no common elements, since if they had, then

$$
\left(n_{1}-\frac{1}{2}-v^{\prime}\right)^{2}=\left(n_{2}-\frac{1}{2}+v^{\prime}\right)^{2},
$$

for $n_{1}, n_{2}$ positive integers. Then, since the expressions in both parentheses are positive, taking square roots on both sides of the last equation gives $n_{1}-n_{2}=2 v$, which is impossible, since $n_{1}-n_{2}$ is integer, while $0<2 v^{\prime}<1$. The value $-1 / 4$ of the coupling corresponds to only one value of $\tilde{v}$, which is $-1 / 2$, and the respective potential has only one sequence of bound-state energies, and eigenfunctions being expressed in terms of the (simple) Laguerre polynomials, as given by (25).

\section{The Three-Dimensional Case}

It is known [10] that the energy eigenfunctions of a spherically symmetric potential $V(r)$ have the form $\psi(r, \theta, \varphi)=R(r) Y_{l}^{m}(\theta, \varphi)$, where $Y_{l}^{m}(\theta, \varphi)$ is a spherical harmonic, with $l, m$ being, respectively, the azimuthal and magnetic quantum numbers, and $R(r)$ is the radial wave function satisfying the radial Schrödinger equation

$$
\frac{d^{2} u(r)}{d r^{2}}+\frac{2 m}{\hbar^{2}}\left(E-\left(V(r)+\frac{l(l+1) \hbar^{2}}{2 m r^{2}}\right)\right) u(r)=0,
$$

where $u(r)=r R(r)$. The function $u(r)$ satisfies homogeneous Dirichlet boundary conditions, i.e. it vanishes at the origin and at infinity, and it behaves as a one-dimensional wave function on the half-line $r \geq 0$. The vanishing of $u(r)$ at infinity is a consequence of the wave function $\psi(r, \theta, \varphi)$ being square integrable, assuming that we consider bound states of the potential $V(r)$. The vanishing of $u(r)$ at the origin results from its vanishing at infinity and the requirement that the radial wave function $R(r)$ must belong to the domain of the radial momentum operator, which is a Hermitian operator ${ }^{2}$. Since the function $u(r)$ satisfies a one-dimensional stationary Schrödinger equation on the half-line, for the effective potential $V(r)+l(l+1) \hbar^{2} / 2 m r^{2}$, and homogeneous Dirichlet boundary conditions, replacing the position $x$ with the distance $r$ and the potential $V(r)$ with the effective potential, the results of section 2 still hold and then, by means of $(22)$, we obtain

$$
V_{\tilde{v} l}(r)=-\frac{8 \varepsilon_{0} \tilde{l}_{0}}{r}+\frac{16(\tilde{v}(\tilde{v}+1)-l(l+1)) \varepsilon_{0} \tilde{l}_{0}^{2}}{r^{2}}
$$

where we used (15) to replace $\hbar^{2} / m$ with $32 \varepsilon_{0} \tilde{l}_{0}^{2}$ in the centrifugal term of the effective potential. The bound-state energies of the potential (26) are then given by (23) and the respective eigenfunctions are given by (24), if $\tilde{v}>-1$ and $\tilde{v} \neq-1 / 2,0,1 / 2, \ldots$, or by $(25)$, if $\tilde{v}=-1 / 2,0,1 / 2 \ldots$

As in the one-dimensional case, the dimensionless coupling $\tilde{v}(\tilde{v}+1)-l(l+1)$ of the inverse square term in (26) is again a quadratic polynomial in $\tilde{v}$ with a global minimum at $-1 / 2$. Thus, in $(-1,0) \backslash\{-1 / 2\}$, the coupling is a two-to-one function mapping the symmetric around $-1 / 2$ values $-1 / 2+v^{\prime}$ and $-1 / 2-v^{\prime}$, where $0<v^{\prime}<1 / 2$, to the same image $v^{\prime 2}-1 / 4-l(l+1)$. Then, every value of the coupling belonging to $(-1 / 4-l(l+1),-l(l+1))$ corresponds to two values of $\tilde{v}$ and thus to two

\footnotetext{
${ }^{2}$ For an analysis of the boundary condition at the origin, see [11].
} 
sequences of bound-state energies (23). For $\tilde{v}=-1 / 2$, the coupling takes its minimum value $-1 / 4-l(l+1)$, and the inverse square term in (26) becomes most attractive, with the respective potential having no additional energy levels. In $[0, \infty)$, the coupling is strictly increasing, and thus one-to-one, and then this interval is mapped to $[-l(l+1), \infty)$ one time. We thus see that apart from all positive values of the coupling, each of its negative values belonging to $[-l(l+1), 0)$ is the image of only one $\tilde{v}$, with the respective potential (26), which has attractive inverse square term, having no additional energy levels (23).

\section{The Kratzer Potential}

For $\tilde{v} \geq l \geq 0$, the coupling $\tilde{v}(\tilde{v}+1)-l(l+1)$, as a function of $\tilde{v}$, is strictly increasing, thus it is one-toone, mapping $[l, \infty)$ to $[0, \infty)$ one time ${ }^{3}$. Then, the resulting pseudo-Coulomb potential (26) can have an arbitrarily repulsive inverse square term, with its bound-state energies being given by only one sequence (23), i.e. there are no additional energy levels. The potential (26) can then be written as

$$
V(r)=-\frac{a}{r}+\frac{b}{r^{2}}
$$

where $a, b$ being arbitrary positive constants ${ }^{4}$. With the parametrization $a=2 D_{e} r_{e}$ and $b=D_{e} r_{e}^{2}$, where $D_{e}, r_{e}$ are positive constants, the potential (27) takes the form

$$
V(r)=-2 D_{e}\left(\frac{r_{e}}{r}-\frac{1}{2}\left(\frac{r}{r}\right)^{2}\right)
$$

If the constants $D_{e}$ and $r_{e}$ are respectively identified as the dissociation energy and the equilibrium internuclear distance in a given diatomic molecule, the potential (28) is the Kratzer potential, which has been studied in several papers [2, 12-15].

Solving (15) for $\tilde{l}_{0}$ and substituting into the expression $\varepsilon_{0} \tilde{l}_{0}$, we obtain

$$
8 \varepsilon_{0} \tilde{l}_{0}=\sqrt{\frac{2 \varepsilon_{0}}{m}} \hbar
$$

Then, setting

$$
8 \varepsilon_{0} \tilde{l}_{0}=2 D_{e} r_{e}
$$

the right-hand sides of the last two equations must be equal, and solving the resulting equation for $\varepsilon_{0}$ yields

We then set

$$
\varepsilon_{0}=\frac{2 m D_{e}^{2} r_{e}^{2}}{\hbar^{2}}
$$

$$
\frac{(\tilde{v}(\tilde{v}+1)-l(l+1)) \hbar^{2}}{2 m}=D_{e} r_{e}^{2}
$$

and solve the quadratic equation for $\tilde{v}$ to obtain

$$
\tilde{v}=-\frac{1}{2}+\sqrt{\left(l+\frac{1}{2}\right)^{2}+\frac{2 m D_{e} r_{e}^{2}}{\hbar^{2}}}
$$

\footnotetext{
${ }^{3}$ The coupling vanishes for $\tilde{v}=l$, and since it is one-to-one for its values in $[0, \infty)$, this is the only value of $\tilde{v}$ for which the coupling vanishes. The resulting potential is then an attractive Coulomb potential that can describe the hydrogen atom.

${ }^{4}$ Using (15), we obtain $\varepsilon_{0} \tilde{l}_{0}=\hbar^{2} / 32 m \tilde{l}_{0}$, and since the constant $\tilde{l}_{0}$ can take any positive value, $\varepsilon_{0} \tilde{l}_{0}$ can also take any positive value. Thus, the Coulomb term $-8 \varepsilon_{0} \tilde{l}_{0} / r$ of the potential (26) can be arbitrarily attractive. Note that although $\varepsilon_{0} \tilde{l}_{0}$ is a function of $\tilde{l}_{0}, \varepsilon_{0} \tilde{l}_{0}^{2}$ is a constant. Then, the change in the Coulomb term of the potential (26) results from the change of $\varepsilon_{0} \tilde{l}_{0}$, while the change in the inverse square term comes from the change in $\tilde{v}$.
} 
since the other root is less than -1 and thus it is rejected. By means of (29) and (31), and using that $\varepsilon_{0} \tilde{l}_{0}^{2}=\hbar^{2} / 32 m$, the potential (26) is transformed into the Kratzer potential (28). Thus, substituting (30) and (32) into (23), we obtain the bound-state energies of the Kratzer potential, which, setting $n^{\prime}=n-1$, take the form

$$
E_{n^{\prime} l}=-\frac{\frac{2 m D_{e}^{2} r_{e}^{2}}{\hbar^{2}}}{\left(n^{\prime}+\frac{1}{2}+\sqrt{\left.\left(l+\frac{1}{2}\right)^{2}+\frac{2 m D_{e} r_{e}^{2}}{\hbar^{2}}\right)^{2}}\right.},
$$

with $n, l=0,1, \ldots$ and $m$ is the reduced mass of the molecule. This is the energy formula for the Kratzer potential found in literature $[13,14,16]$.

\section{Weakly Attractive Inverse Square Term}

The Kratzer potential corresponds to the positive values of the coupling $\tilde{v}(\tilde{v}+1)-l(l+1)$, for which the potential (26) has no additional energy levels. The same happens when the coupling takes negative values in $[-l(l+1), 0)$, for $l \neq 0$. In this case, we set

$$
\tilde{v}(\tilde{v}+1)-l(l+1)=v^{\prime}
$$

with $-l(l+1) \leq v^{\prime}<0$, and then we solve the quadratic equation for $\tilde{\boldsymbol{v}}$ to obtain

$$
\tilde{v}=-\frac{1}{2}+\sqrt{\left(l+\frac{1}{2}\right)^{2}+v^{\prime}}
$$

since the other root is equal to or less $\operatorname{than}^{5}-1$ and thus it is rejected. Then, by means of (33), the potential (26) reads

$$
V_{v^{\prime}}(r)=-\frac{8 \varepsilon_{0} \tilde{l}_{0}}{r}+\frac{16 v^{\prime} \varepsilon_{0} \tilde{l}_{0}^{2}}{r^{2}}
$$

with both the Coulomb and the inverse square term being attractive $\left(v^{\prime}<0\right)$. Substituting (34) into (23), we obtain the bound-state energies of the previous potential, which, setting again $n^{\prime}=n-1$, take the form

$$
E_{n^{\prime} l}=-\frac{\varepsilon_{0}}{\left(n^{\prime}+\frac{1}{2}+\sqrt{\left(l+\frac{1}{2}\right)^{2}+v^{\prime}}\right)^{2}}
$$

with $n^{\prime}, l=0,1, \ldots$

Contrary to the previous case, when the coupling $\tilde{v}(\tilde{v}+1)-l(l+1)$ takes values in $(-1 / 4-l(l+1)$, $-l(l+1))$, it is a two-to-one function in $\tilde{v}$ and the potential (26) has then additional energy levels. Indeed, introducing again the parameter $v^{\prime}$ through $(33)$, with $-1 / 4-l(l+1)<v^{\prime}<-l(l+1)$, and solving the quadratic equation for $\tilde{v}$, both roots are now accepted, since they are both greater than -1 , and thus

$$
\tilde{v}=-\frac{1}{2} \pm \sqrt{\left(l+\frac{1}{2}\right)^{2}+v^{\prime}}
$$

${ }^{5}$ Since $v^{\prime} \geq-l(l+1)$, we have $l^{2}+l+v^{\prime} \geq 0 \Rightarrow 4\left(l^{2}+l+v^{\prime}\right) \geq 0 \Rightarrow 4\left(l^{2}+l+\frac{1}{4}+v^{\prime}\right) \geq 1 \Rightarrow 4\left(\left(l+\frac{1}{2}\right)^{2}+v^{\prime}\right) \geq 1 \Rightarrow$ $\Rightarrow \sqrt{\left(l+\frac{1}{2}\right)^{2}+v^{\prime}} \geq \frac{1}{2} \Rightarrow-\sqrt{\left(l+\frac{1}{2}\right)^{2}+v^{\prime}} \leq-\frac{1}{2} \Rightarrow-\frac{1}{2}-\sqrt{\left(l+\frac{1}{2}\right)^{2}+v^{\prime}} \leq-1$ 
Then, the potential (26), which again takes the form

$$
V_{v^{\prime}}(r)=-\frac{8 \varepsilon_{0} \tilde{l}_{0}}{r}+\frac{16 v^{\prime} \varepsilon_{0} \tilde{l}_{0}^{2}}{r^{2}}
$$

with both terms being attractive $\left(v^{\prime}<0\right)$, has the bound-state energies

$$
E_{n^{\prime} l}=-\frac{\varepsilon_{0}}{\left(n^{\prime}+\frac{1}{2} \pm \sqrt{\left(l+\frac{1}{2}\right)^{2}+v^{\prime}}\right)^{2}},
$$

where we again set $n^{\prime}=n-1$, and thus $n^{\prime}, l=0,1, \ldots$

Finally, when the coupling $\tilde{v}(\tilde{v}+1)-l(l+1)$ takes its minimum value, which is $-1 / 4-l(l+1)$ and corresponds to $\tilde{v}=-1 / 2$, the potential $(26) \operatorname{reads}^{6}$

$$
V_{l}(r)=-\frac{8 \varepsilon_{0} \tilde{l}_{0}}{r}-\frac{4(2 l+1)^{2} \varepsilon_{0} \tilde{l}_{0}^{2}}{r^{2}}, l=0,1, \ldots
$$

The previous potential has no additional energy levels and, in line with (23), its bound-state energies are given by

$$
E_{n^{\prime}}=-\frac{\varepsilon_{0}}{\left(n^{\prime}+\frac{1}{2}\right)^{2}},
$$

where we again set $n^{\prime}=n-1$, and thus $n^{\prime}=0,1, \ldots$ As in the one-dimensional case, the respective eigenfunctions are given in terms of the (simple) Laguerre polynomials.

\section{Relaxation of the Boundary Condition at the Origin and Instability}

We assume that, instead of vanishing, the function $u(r)$ can take a constant, non-zero value at the origin. This is the slightest relaxation of the boundary condition at the origin, and since $R(r)=u(r) / r$, it corresponds to a $1 / r$ behaviour of the radial wave function at the origin. For $l=0$, i.e. if the particle has no orbital angular momentum, the radial Schrödinger equation takes the form of the onedimensional Schrödinger equation for the examined pseudo-Coulomb potential on the half-line, with the solution $u(r)$ being the one-dimensional wave function $\psi(x)$, which can then take a constant, non-zero value at zero. Then, from the analysis in section 2 , we see that the parameter $\tilde{v}$ can also take the value -1 and as a consequence, the coupling $\tilde{v}(\tilde{v}+1)$ of the inverse square term of the potential now vanishes not only for $\tilde{v}=0$, but also for $\tilde{v}=-1$. Then, the respective attractive Coulomb potential has, in line with (23), the following two sequences of bound-state energies

$$
E_{n_{1}}=-\frac{\varepsilon_{0}}{n_{1}^{2}}, E_{n_{2}}=-\frac{\varepsilon_{0}}{\left(n_{2}-1\right)^{2}},
$$

where $n_{1}, n_{2}=1,2, \ldots$. The second sequence contains all the energies of the first sequence, since for $n_{2}=$ $n_{1}+1 \geq 2$ the two sequences coincide, and one extra energy, which results for $n_{2}=1$ and it is minus infinity. According to [1], such an energy, which is the ground-state energy for the respective Coulomb potential, corresponds to an unstable state, where the particle falls to the centre.

\section{Concluding Remarks}

We have presented the bound-state solutions of the stationary Schrödinger equation in one and three dimensions, for a general pseudo-Coulomb potential containing an attractive Coulomb term and an inverse square term with coupling not less than a negative critical value, below which the particle is

${ }^{6}-\frac{1}{4}-l(l+1)=-\frac{1}{4}(2 l+1)^{2}$ 
unstable and falls to the centre. We have shown that in a subset of its domain, the inverse-square-term coupling is a two-to-one mapping and, as a consequence, the respective potentials, all having attractive inverse square term, are endowed with a second sequence of bound-state energies. Finally, we have demonstrated that the slightest relaxation in the boundary condition for the radial wave function at the origin results in one additional, unbounded below, energy for the respective Coulomb potential and thus in instability of the hydrogen atom.

\section{References}

1. L. D. Landau and E. M. Lifshitz, Quantum Mechanics. Pergamon Press Ltd., Second Edition, 1965, pp. 113-115.

2. A. Kratzer, "Die ultraroten Rotationsspektren der Halogenwasserstoffe", Z. Phys. 3, 289 (1920); E. Fues, "Zur Intensität der Bandenlinien und des Affinitätsspektrums zweiatomiger Moleküle", Ann. Phys. 80, 281 (1926).

3. R. J. Le Roy and R. B. Bernstein, "Dissociation Energy and Long-Range Potential of Diatomic Molecules from Vibrational Spacings of Higher Levels", J. Chem. Phys. 52, 3869 (1970).

4. S. Frish, Optical Spectra of Atoms. Fizmatgiz, Moscow, 1963.

5. Teimuraz Nadareishvili and Anzor Khelashvili, "Pragmatic SAE procedure in the Schrodinger equation for the inverse-square-like potentials", Available: https://arxiv.org/abs/1209.2864

6. Mary L. Boas, Mathematical Methods in the Physical Sciences. John Wiley \& Sons, Inc., Third Edition, 2006, pp. 605-606.

7. Alexander V. Turbiner, "One-dimensional quasi-exactly solvable Schrödinger equations", Phys. Rep. 642, 1-71 (2016).

8. Arfken, Weber, and Harris, Mathematical Methods for Physicists. Elsevier Inc., Seventh Edition, 2013, p. 892.

9. Michael Martin Nieto, "Hydrogen atom and relativistic pi-mesic atom in N-space dimensions", Am. J. Phys. 47, 1067 (1979).

10. David J. Griffiths, Introduction to Quantum Mechanics. Pearson Education, Inc., Second Edition, 2005, pp. 131141.

11. Anzor A. Khelashvili and Teimuraz P. Nadareishvili, "What is the boundary condition for the radial wave function of the Schrödinger equation?", Am. J. Phys. 79 (6), 668-671 (2011).

12. Djamil Bouaziz, "Kratzer's molecular potential in quantum mechanics with a generalized uncertainty principle", Ann. Phys. 355, 269-281 (2015).

13. O. Bayrak, I. Boztosun, and H. Ciftci, "Exact Analytical Solutions to the Kratzer Potential by the Asymptotic Iteration Method", Int. J. Quantum Chem. 107 (3), 540-544 (2007).

14. Sameer M. Ikhdair and Ramazan Sever, "Exact quantization rule to the Kratzer-type potentials: an application to the diatomic molecules", J. Math. Chem. 45, 1137 (2009).

15. Mohammad R Setare and Ebrahim Karimi, "Algebraic approach to the Kratzer potential", Phys. Scr. 75, 90-93 (2007).

16. Mohammad Reza Pahlavani, Theoretical Concepts of Quantum Mechanics. InTech, 2012, p. 242.

17. Spiros Konstantogiannis, "Polynomial and Non-Polynomial Terminating Series Solutions to the Associated Laguerre Differential Equation", ResearchGate, DOI: 10.13140/RG.2.2.24904.98569.

\section{Appendix}

Theorem 1 The non-terminating Frobenius solutions of the associated Laguerre equation behave as $\exp (x)$ at infinity.

Proof: Considering the associated Laguerre equation

$$
x y^{\prime \prime}(x)+(v+1-x) y^{\prime}(x)+\lambda y(x)=0, \quad v, \lambda \in \mathbb{R},
$$

we see that zero is a regular singular point. Then, in line with the Fuchs' theorem [6], let

$$
y(x)=\sum_{n=0}^{\infty} c_{n} x^{n+r}, c_{0} \neq 0,
$$

be a Frobenius solution to the equation in the positive region near zero. Differentiating the previous series term by term, twice, we have, respectively, 


$$
y^{\prime}(x)=\sum_{n=0}^{\infty}(n+r) c_{n} x^{n+r-1}, y^{\prime \prime}(x)=\sum_{n=0}^{\infty}(n+r)(n+r-1) c_{n} x^{n+r-2} .
$$

Substituting the expressions of $y(x)$ and its first and second derivative into the associated Laguerre equation, shifting the summation indices appropriately and collecting coefficients of the same powers of $x$, we end up to the equation

$$
r(r+v) c_{0} x^{r-1}+\sum_{n=1}^{\infty}\left((n+r)(n+r+v) c_{n}-(n+r-(\lambda+1)) c_{n-1}\right) x^{n+r-1}=0,
$$

from which we obtain, since $c_{0} \neq 0$,

$$
r(r+v)=0
$$

which is the indicial equation giving the exponents $r=0,-v$, and the recurrence relation

$$
(n+r)(n+r+v) c_{n}-(n+r-(\lambda+1)) c_{n-1}=0, n=1,2, \ldots
$$

To solve the recurrence relation for $c_{n}$, the coefficient $(n+r)(n+r+v)$ must be non-zero. If $r=0$, this coefficient becomes $n(n+v)$, and thus it is non-zero iff $v \neq-1,-2, \ldots$. If $r=-v$, the said coefficient becomes $(n-v) n$, and thus it is non-zero iff $v \neq 1,2, \ldots$ Then, iff $v$ is neither positive nor negative integer, the recurrence relation is solved for both exponents, thus, in this case, the associated Laguerre equation has two linearly independent Frobenius solutions, while if $v$ is either positive or negative integer, the recurrence relation is solved only for one of the two exponents, since for the other exponent it yields an undetermined coefficient $c_{n}$ and then all subsequent coefficients are also undetermined, and thus, in this case, the equation has only one Frobenius solution. If $v$ vanishes, the indicial equation has zero as double root, and the recurrence relation takes the form

$$
n^{2} c_{n}-(n-(\lambda+1)) c_{n-1}=0, n=1,2, \ldots,
$$

which can be solved for $c_{n}$, since $n \neq 0$. Thus, we have again only one Frobenius solution. Therefore, if $v$ is not integer, the associated Laguerre equation has two Frobenius solutions, while if $v$ is integer, it has one Frobenius solution and, in line with the Fuchs' theorem, the other linearly independent solution has a logarithmic part. Then, solving the initial recurrence relation for $c_{n}$ yields

$$
c_{n}=\frac{n+r-(\lambda+1)}{(n+r)(n+r+v)} c_{n-1}, n=1,2, \ldots, \quad c_{0} \neq 0
$$

Thus, the respective Frobenius series does not terminate iff $n+r-(\lambda+1) \neq 0$, for every $n=1,2, \ldots$. Then, by the ratio test, we find that the series converges absolutely for all positive $x$. Since the solution holds for $x \rightarrow \infty$, we can examine its asymptotic behaviour, which is determined by the coefficients of the high powers of $x$, i.e. by the coefficients $c_{n}$ with big $n$. From the previous recurrence relation, we see that, for big $n$,

$$
\frac{c_{n+1}}{c_{n}} \simeq \frac{n}{n^{2}}=\frac{1}{n} \simeq \frac{1}{n+1},
$$

thus

$$
c_{n+1} \simeq \frac{c_{n}}{n+1}
$$

The previous recurrence relation is easily solved to give

$$
c_{n+1}=\frac{1}{(n+1) !} c_{0},
$$

which are the coefficients of the series expansion of $c_{0} \exp (x)$ about zero. Therefore, the non-terminating Frobenius solutions of the associated Laguerre equation behave as $\exp (x)$ at infinity.

Theorem 2 Considering the associated Laguerre equation

$$
x y^{\prime \prime}(x)+(v+1-x) y^{\prime}(x)+\lambda y(x)=0, \quad v, \lambda \in \mathbb{R},
$$

we have the following cases:

1. If $v$ is not integer and $\lambda=0,1, \ldots$, the associated Laguerre equation has a polynomial solution 


$$
y(x)=\sum_{n=0}^{\lambda} c_{n} x^{n}, \quad c_{n+1}=\frac{n-\lambda}{(v+n+1)(n+1)} c_{n}, n=0,1, \ldots, \lambda-1
$$

If $\lambda=0$, the polynomial is a non-zero constant.

2. If $v$ is not integer and $\lambda=-v,-v+1, \ldots$, the associated Laguerre equation has a terminating series solution, which is not a polynomial and has the form

$$
y(x)=\sum_{n=0}^{\lambda+v} c_{n} x^{n-v}, c_{n+1}=\frac{n-(\lambda+v)}{(n-v+1)(n+1)} c_{n}, n=0,1, \ldots, \lambda+v-1
$$

If $\lambda=-v$, then $y(x) \sim x^{-v}$. The solution holds in the region $x>0$, while in the region $x<0$, it is derived similarly.

3. If $v, \lambda=0,1, \ldots$, the associated Laguerre equation has a polynomial solution, which is, up to a multiplicative real constant, the associated Laguerre polynomial $L_{\lambda}^{\nu}(x)$ of degree $\lambda$ with parameter $v$, which is given by

$$
L_{\lambda}^{v}(x)=\sum_{n=0}^{\lambda} \frac{(-1)^{n}}{n !}\left(\begin{array}{c}
\lambda+v \\
\lambda-n
\end{array}\right) x^{n},\left(\begin{array}{c}
\lambda+v \\
\lambda-n
\end{array}\right)=\frac{(\lambda+v) !}{(\lambda-n) !(v+n) !},
$$

In the special case where $v=0$, the associated Laguerre equation reduces to the Laguerre equation and the associated Laguerre polynomials reduce to the (simple) Laguerre polynomials. As a consequence, the Laguerre equation has polynomial solution iff $\lambda=0,1, \ldots$, and the polynomial solution is, up to a multiplicative real constant, the Laguerre polynomial $L_{\lambda}(x)$ of degree $\lambda$.

4. If $v$ is negative integer and $\lambda=-v,-v+1, \ldots$, the associated Laguerre equation has a polynomial solution, which is, up to a multiplicative real constant, equal to $x^{-v} L_{\lambda+v}^{-v}(x)$. The solution holds in the region $x>0$, while in the region $x<0$, it is derived similarly. The associated Laguerre polynomial $L_{\lambda+v}^{-v}(x)$ is of degree $\lambda+v$, thus the previous solution is a polynomial of degree $\lambda$. If $\lambda=-v$, the associated Laguerre polynomial is equal to 1 and the polynomial solution is, up to a multiplicative real constant, equal to $x^{-v}$.

Proof: The proof is straightforward and is done by using the Fuchs' theorem in the way we outlined in the previous theorem. For the details, the reader may refer to [17]. 